\title{
A Complete Public-Key Cryptosystem
}

\author{
Dima Grigoriev \\ IRMAR, Université de Rennes, \\ Campus de Beaulieu, 35042 Rennes, France \\ http://perso.univ-rennes1.fr/dmitry.grigoryev/
}

Edward A. Hirsch*

Steklov Institute of Mathematics at St.Petersburg, 27 Fontanka, 191023 St.Petersburg, Russia

http://logic.pdmi.ras.ru/ hirsch/

Konstantin Pervyshev

University of California, San Diego, USA

Received: January 11, 2008

\begin{abstract}
We present a cryptosystem which is complete for the class of probabilistic public-key cryptosystems with bounded error. Besides traditional encryption schemes such as RSA and El Gamal and probabilistic encryption of Goldwasser and Micali, this class contains also Ajtai-Dwork and NTRU cryptosystems. The latter two make errors with a small positive probability.
\end{abstract}

\section{Introduction}

Reductions between computational problems have great meaning for complexity theory. In particular, reduction is a natural tool for establishing computational hardness of various algorithmic problems. With reductions, one can prove that some problem is at least as hard as another one. Furthermore, for some classes of problems, it is possible to find a complete problem, which is at least as hard as any other problem in the class.

The same idea can be applied to cryptography, where problems of breaking cryptographic primitives are considered. The notion of a reduction and the notion of completeness may be adapted to this cryptographic setting. In particular, they may help to find the hardest to break implementations of cryptographic primitives. If a complete public-key cryptosystem $\mathcal{C}$ exists, one could argue that under the assumption that secure public-key cryptography is possible at all, the cryptosystem $\mathcal{C}$ is secure.

There is a number of important cryptographic primitives such as, to name a few, one-way functions and public-key cryptosystems. In [14], Levin presented a universal (complete) one-way function. Informally speaking, it is a polynomial-time

*Supported in part by Dynasty Fellowship, RFBR grant 08-01-00640-a, and the President of Russia grant for leading scientific schools support NSh-4392.2008.1.

ISSN 1867-1144 / \$2.50 C Heldermann Verlag 
computable function which is the hardest to invert (read "to break") among all polynomial-time computable functions. Therefore, if hard-to-invert polynomialtime functions exist, then Levin's function is one of them. Readers are referred to [7, Section 2.4.1] for a more detailed discussion. Actually, the idea can be traced back to Levin's optimal algorithm for NP-complete problems. The same idea was used later to construct a universal learning algorithm [8]. Surprisingly, complete public-key encryption schemes remained unknown.

In 2005, Harnik et al. [9] came up with a notion of robust combiners which, in the case of public-key encryption schemes, led to a construction of a complete scheme.

The paper [9] is a conference proceedings version, and the proof that the construction indeed works is only sketched. We give a detailed presentation of the construction of a complete public-key cryptosystem and the proof of its completeness. It follows a preprint version ${ }^{1}$ of our paper; at the time our preprint appeared, we were not aware of Harnik et al.'s work.

\subsection{Probabilistic Encryption with Bounded Error}

We look for a complete public-key encryption scheme in the class of probabilistic encryption schemes with bounded error rather than in the class of all "perfect" cryptosystems (that do not have encryption/decryption errors). The fact that we consider "imperfect" probabilistic encryption schemes makes the result even more interesting. We present a public-key cryptosystem which is complete for the class that contains all "traditional" public-key encryption schemes as well as probabilistic encryption of Goldwasser and Micali [6], one of the first encryptions schemes, Ajtai-Dwork cryptosystem [1], which is based on lattice problems, and NTRU [11]. The latter two allow a small positive probability of error during the encryption and decryption of a message.

It is known that error probability of probabilistic cryptosystems can be easily reduced down to a negligible one $[5,2]$.

\subsection{Reductions}

In order to formalize the notion of "the hardest-to-break" cryptographic primitive, we use reductions. A cryptographic primitive $S_{1}$ is reducible to another primitive $S_{2}$, iff there exists a probabilistic oracle procedure $R$ (called a reduction) that, given a (probabilistic) oracle $A$ that breaks $S_{2}$, breaks $S_{1}$. As an illustration, every one-way function can be inverted by a polynomial-time algorithm given an access to a probabilistic oracle for inverting Levin's function with considerable probability. Therefore, one can call Levin's function a complete one-way function. also believed to be unsolvable in polynomial time, problems known to be NPcomplete are less likely to have efficient solutions. In the similar way, it makes

\footnotetext{
${ }^{1}$ Electronic Colloquium on Computational Complexity, ECCC TR06-046, http://eccc.hpiweb.de/eccc-reports/2006/TR06-046/index.html
} 
sense to use complete cryptographic primitives as they are less likely to be breakable by polynomial-time adversaries.

\subsection{Main Result}

Levin's universal one-way function [14] implies universal constructions of many other one-way-functions-based cryptographic primitives such as digital signature, private-key encryption and MAC (cf. the discussion in [9]). However, it does not imply universal public-key cryptosystem, because the existence of one-way functions does not suffice for public-key cryptography. Levin's idea does not work here either: In order to construct a complete cryptosystem, we have to overcome the difficulty that not every triple of Turing machines corresponds to a correct encryption scheme (it may be just undecryptable). developed only recently [2].

We stress that our encryption scheme is secure only for huge values of its security parameter. For such values, key generation, encryption and decryption algorithms, which are polynomial-time in the security parameter, require a huge amount of time and communication. Therefore it is highly interesting to find practical encryption schemes that are complete.

\subsection{Related Work}

In complexity theory, the existence of a complete language for some class of languages is closely related to the existence of a time hierarchy in the class: namely, both complete languages and hierarchy theorems are known for "syntactic" (i.e., efficiently enumerable) classes, but are hard to devise for other ("semantic") classes. An interesting paper of Fortnow and Santhanam [3] shows that there exists a time hierarchy for heuristic probabilistic algorithms with two-sided error (for which no complete problems are known). Such algorithms are permitted to violate the error bound on a small fraction of inputs.

An important ingredient of our proof is the amplification of cryptosystem correctness, i.e. a procedure that reduces the error probability of a given cryptosystem while moderately worsening its security. Such correctness amplification technique is known from the work of Dwork, Naor and Reingold [2]. For more discussion on this topic, see papers by Holenstein and Renner $[12,10]$.

\section{Definitions}

Definition 2.1. A public key encryption scheme $S$ consists of three probabilistic worst-case polynomial-time algorithms $(G, E, D)$, for key generation, encryption and decryption respectively.

Key generation algorithm $G$ is given a security parameter $1^{n}$ as input, and outputs the public key and secret key pair $(p k, s k) \leftarrow G\left(1^{n}\right)$. Encryption algorithm $E$ takes as input a public key $p k$, a one-bit plaintext message $m$ and produces a ciphertext $M=E_{p k}(m)$. Finally, decryption algorithm $D$ takes as input a secret 
key $s k$ and a ciphertext. The output of $D$ is a message $m^{\prime}=D_{s k}(M)$, which may fail to equal the original message $m$. For more details, see [4].

Definition 2.2. A public key encryption scheme is $\delta(n)$-correct, iff for all sufficiently large security parameters $n$, for any one-bit message $m \in\{0,1\}$,

$$
\operatorname{Pr}\left[D_{s k}\left(E_{p k}(m)\right)=m\right] \geq \delta(n) \text { for }(p k, s k) \leftarrow G\left(1^{n}\right),
$$

where probability is taken over randomness of algorithms $G, E$ and $D$.

Definition 2.3. A probabilistic black-box $A \epsilon(n)$-breaks an encryption scheme $(G, E, D)$, if for infinitely many security parameters $n$,

$$
\operatorname{Pr}\left[A_{p k}\left(1^{n}, E_{p k}(m)\right)=m\right] \geq \epsilon(n) \text { for }(p k, s k) \leftarrow G\left(1^{n}\right),
$$

where probability is taken over uniform selection of a one-bit message $m$ and over randomness of algorithms $G, E$ and of black-box $A$.

Since we consider public-key encryption schemes that encode one-bit messages, it makes sense to consider only those $\epsilon(n)$ and $\delta(n)$ that are greater than $1 / 2$. Also, note that Definition 2.2 implies that knowing private key sk one can $\delta(n)$-break the encryption scheme.

We could have defined $\delta(n)$-correct encryption schemes in a different way, so that the probability in the definition is also taken over randomly chosen messages $m \in\{0,1\}$ like in Definition 2.3. However, Definition 2.2 that we use makes our construction of a public-key encryption scheme a little bit easier.

Definition 2.4. A probabilistic black-box $A$ breaks an encryption scheme $(G, E$, $D)$, if it $(1 / 2+1 / p(n))$-breaks the encryption scheme for some polynomial $p(n)$.

An encryption scheme $(G, E, D)$ is secure, if no probabilistic polynomial-time Turing machine breaks $(G, E, D)$.

Some encryption schemes may be harder to break than others. So let us define a reduction between a pair of encryption schemes similarly to that between two languages. In some sense, the notion of a break of a cryptosystem is an analogue of recognition of a language in complexity theory.

Definition 2.5. An encryption scheme $\left(G_{1}, E_{1}, D_{1}\right)$ is reducible to an encryption scheme $\left(G_{2}, E_{2}, D_{2}\right)$, if there exists a probabilistic polynomial time oracle machine $R$, such that for any probabilistic black-box $A$ that breaks $\left(G_{2}, E_{2}, D_{2}\right), R^{A}$ breaks $\left(G_{1}, E_{1}, D_{1}\right) .^{2}$

Obviously, if encryption scheme $\left(G_{1}, E_{1}, D_{1}\right)$ is secure, then $\left(G_{2}, E_{2}, D_{2}\right)$ is also secure. Thus, it makes sense to find a complete encryption scheme, a one to which any other encryption scheme, secure or not, is reducible.

${ }^{2}$ Note that, by Definition $2.3, R^{A}$ is provided with a security parameter $1^{n}$ as well as with a public key $p k$. 
Proposition 2.6. Reductions are transitive.

\section{Proof strategy}

In the construction of a complete public key encryption scheme (which is the hardest to break among all encryption schemes), we employ a natural idea of combining all possible public-key encryption schemes $\left(G_{i}, E_{i}, D_{i}\right)_{i \in \mathbb{N}}$, in order to achieve the maximal possible level of security. For a given security parameter $n$, we combine the first $n$ of encryption schemes in a way that their combination is secure if and only if at least one of them is secure.

To encrypt a message $m \in\{0,1\}$, our complete public key encryption scheme $\mathcal{S}=(\mathcal{G}, \mathcal{E}, \mathcal{D})$ performs as follows. First, key generator $\mathcal{G}$ on input $1^{n}$ obtains pairs of public and private keys $\left(p k_{1}, s k_{1}\right) \leftarrow G_{1}\left(1^{n}\right), \ldots\left(p k_{n}, s k_{n}\right) \leftarrow G_{n}\left(1^{n}\right)$. As we will see in Lemma 6.1, w.l.o.g. key generators $G_{i}$ work in time, say, $n^{4}$.

Then, encryption algorithm $\mathcal{E}$ selects $x_{1}, \ldots x_{n} \in_{R}\{0,1\}$ (i.e. independently and uniformly at random from $\{0,1\})$ and produces a codeword $\left(E_{1, p k_{1}}\left(x_{1}\right), \ldots\right.$, $\left.E_{n, p k_{n}}\left(x_{n}\right), x_{1} \oplus \ldots \oplus x_{n} \oplus m\right)$. Evidently, if at least one encryption $E_{i}$ is secure, then no adversary has noticeable advantage over random guessing in learning $x_{i}$ from $E_{i, p k_{i}}\left(x_{i}\right)$, thus in learning $m$ from $x_{1} \oplus \ldots \oplus x_{n} \oplus m$, even if all other encryption schemes are insecure.

In order to extract the message $m$ from $x_{1} \oplus \ldots \oplus x_{n} \oplus m$, having our private keys $\left(s k_{1}, \ldots s k_{n}\right)$, we need to recover all $x_{j}$ from $E_{j, p k_{j}}\left(x_{j}\right)$. However, most of encryption schemes are probably not correct in the sense that $D_{j, s k_{j}}\left(E_{j, p k_{j}}\left(x_{j}\right)\right)$ is not necessarily equal to $x_{j}$. We overcome this difficulty by using only those encryption schemes that are correct in a certain sense. And this is exactly the place where we have to allow encryption/decryption errors.

Although it is impossible to test a given encryption scheme for $100 \%$ correctness, we are able to determine with high confidence whether a given scheme is almost correct. So we select those encryption schemes that pass our test for "almost correctness" and combine them in our construction. Since the errors in several almost correct schemes may accumulate and increase, we employ correctness amplification technique [2], the last ingredient of our proof.

Definition 3.1. We denote the class of all 2/3-correct public key encryption schemes (every algorithm of which runs in probabilistic polynomial time) with PKCS.

Sometimes, a key generation algorithm is allowed to run in expected polynomial time [4, Section 7.1]. However, since we allow encryption schemes to fail with positive probability, these two possible definitions are equivalent.

Notice that we do not include any requirement on security into this definition. Therefore some schemes from PKCS may be much more easier (sometimes trivial) to break than others. Our goal is exactly to find the scheme that is the 
hardest-to-break in this class.

The choice of the constant $2 / 3$ in the definition is arbitrary because it is possible to efficiently amplify correctness of an encryption scheme while preserving its security. In fact, we could have defined PKCS as a class of encryption schemes that are $\left(1-\frac{1}{p(n)}\right)$-correct for any fixed polynomial $p(n)$.

The remaining part of the paper constitutes a proof of the following theorem.

Theorem 3.2. There exists a complete PKCS.

\section{Correctness Test}

In order to distinguish for a given security parameter $n$ between those encryption schemes that are $(\delta+\Delta)$-correct and those that are less than $(\delta-\Delta)$-correct, we will use the straightforward test given below:

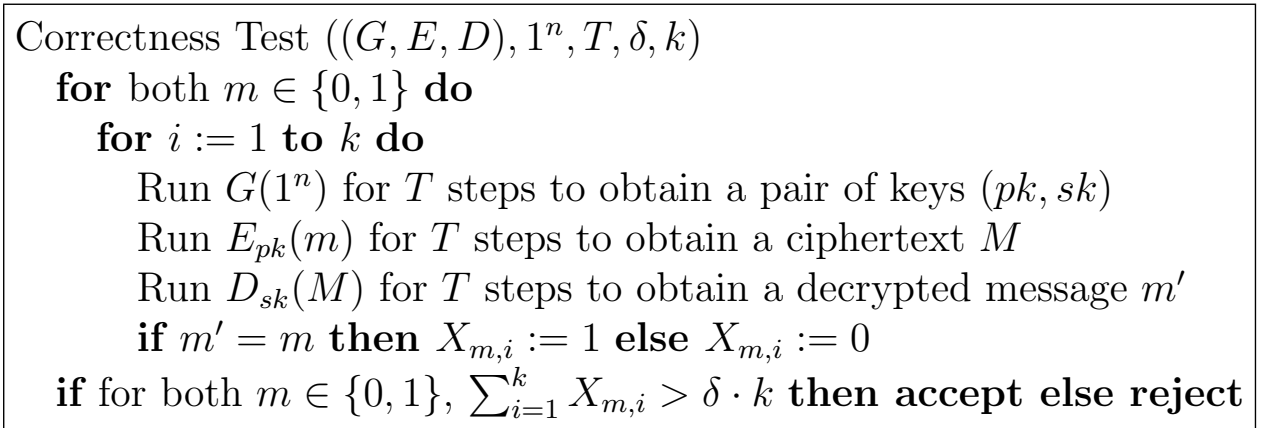

Figure 4.1

In this test, if one of the algorithms $G, E$, and $D$ wants to work for more than $T$ steps, it is stopped after allowed $T$ execution steps, and its output tape by that moment is considered as the result of the execution.

Proposition 4.1 (Chernoff bound). Let $X_{1}, X_{2}, \ldots, X_{n}$ be independent Poisson trials with $\operatorname{Pr}\left[X_{i}=1\right]=p_{i}$ and $\operatorname{Pr}\left[X_{i}=0\right]=1-p_{i}$. Then if $X$ is the sum of $X_{i}$ and if $\mu$ is $\mathbb{E}[X]$, for any $\lambda, 0<\lambda \leq \mu$ :

$$
\operatorname{Pr}[X \leq \mu-\lambda]<e^{-\lambda^{2} / 2 n}, \quad \operatorname{Pr}[X \geq \mu+\lambda]<e^{-\lambda^{2} / 2 n} .
$$

The following proposition can be proved using the Chernoff and union bounds:

Proposition 4.2. For a given security parameter $n$, every $(\delta+\Delta)$-correct encryption scheme, every algorithm of which runs in time $T$, passes the test almost for sure, failing with probability less than $2 e^{-\frac{\Delta^{2} \cdot k}{2}}$. At the same time, if the encryption scheme, every algorithm of which is restricted to run no more than time $T$, is less than $(\delta-\Delta)$-correct, then it passes the test with probability at most $e^{-\frac{\Delta^{2} \cdot k}{2}}$. 
Consequently, we can distinguish in probabilistic polynomial time with high confidence those encryption schemes that are, say, $\left(1-\frac{1}{12 n}\right)$-correct from those that are not $\left(1-\frac{1}{4 n}\right)$-correct. Thus, if we have $n$ encryption schemes $S_{i}, 1 \leq i \leq n$, and combine into a new encryption scheme (Section 6.1) only those of them that pass the test, we get at least $2 / 3$-correct encryption scheme (by union bound). It remains to show that every scheme $S_{i} \in \mathbf{P K C S}$, which is $2 / 3$-correct, is reducible to some $\left(1-\frac{1}{12 n}\right)$-correct encryption scheme, that is to amplify the correctness of encryption schemes (cf. [2]).

\section{Correctness Amplification}

Assume our encryption scheme $(G, E, D)$ is secure and $(1 / 2+\delta(n))$-correct. Our aim is to improve its reliability while moderately worsening its security. The recent work by Dwork, Naor and Reingold [2] provides a way to achieve this. However, we present an amplification scheme here because we need to prove that there is a reduction (in our sense) from a weakly correct encryption scheme to an encryption scheme with amplified correctness.

Definition 5.1. Let $S^{k(n)}=(G, E, D)^{k(n)}$ be the encryption scheme obtained from encryption scheme $S=(G, E, D)$ as follows. The key generation algorithm $G^{k(n)}$ on input $1^{n}$ runs $k(n)$ copies of $G\left(1^{n}\right)$ with independent sources of randomness to obtain pairs of keys $\left(p k_{1}, s k_{1}\right), \ldots,\left(p k_{k(n)}, s k_{k(n)}\right)$. The encryption algorithm $E^{k(n)}$, provided a public key $p k_{1}, \ldots, p k_{k(n)}$ and a message $m$, runs $k(n)$ copies of $E\left(m, p k_{i}\right)$ (one copy per one value of $i$ ) with independent sources of randomness to obtain codewords $M_{1}, \ldots, M_{k(n)}$. Finally, the decryption algorithm $D^{k(n)}$, provided a private key $s k_{1}, \ldots, s k_{k(n)}$ and an encoded message $M_{1}, \ldots, M_{k(n)}$, runs $k(n)$ copies of $D\left(M_{i}, s k_{i}\right)$ with independent sources of randomness to obtain codewords $m_{1}^{\prime}, \ldots, m_{k(n)}^{\prime}$ and outputs their majority value (we assume that $k(n)$ is odd).

The next two lemmas are close to [2, Lemma 3]. In the first lemma, which is responsible for the security aspect of the construction, we prove that, for any polynomial $k(n),(G, E, D)$ is reducible to $(G, E, D)^{k(n)}$. Then, in the second lemma, responsible for the correctness, we show that the probability of error of $(G, E, D)^{k(n)}$ is exponentially smaller than the probability of error of the original cryptosystem.

Lemma 5.2. Every encryption scheme $S=(G, E, D)$ is reducible via some reduction $R$ to the encryption scheme $S^{k(n)}=(G, E, D)^{k(n)}$. Furthermore, if some black-box $A(1 / 2+\epsilon(n))$-breaks $(G, E, D)^{k(n)}$, then $R^{A}(1 / 2+\epsilon(n) / k(n))$-breaks $(G, E, D)$.

Proof. Assume an adversary $A$ breaks $S^{k(n)}$ with probability greater than $1 / 2+$ $\epsilon(n)$ for infinitely many security parameters $n$. Let us construct an adversary $R$ that breaks $S$ with probability greater than $1 / 2+\epsilon(n) / k(n)$ for the same set of 
security parameters $n$ using adversary $A$ as a black-box.

Our reduction $R$ (with oracle $A$ ) on input composed of a public key $p k$, a security parameter $1^{n}$, and a codeword $M=E_{p k}(m)$ works as follows:

1. Choose $i$ from $\{1,2, \ldots, k\}$ uniformly at random. ${ }^{3}$

2. Generate keys $\left(p k_{1}, s k_{1}\right), \ldots,\left(p k_{k}, s k_{k}\right) \leftarrow G^{k}\left(1^{n}\right)$ (actually, we need only the public keys for our reduction).

3. Invoke encryption $\left(M_{1}, \ldots, M_{i-1}\right) \leftarrow E_{\left(p k_{1}, \ldots p k_{i-1}\right)}^{i-1}(0)$.

4. Invoke encryption $\left(M_{i+1}, \ldots M_{k}\right) \leftarrow E_{\left(p k_{i+1}, \ldots p k_{k}\right)}^{k-i}(1)$.

5. Output $A\left(M_{1}, \ldots M_{i-1}, M, M_{i+1}, \ldots M_{k}\right)$.

Note that all invocations of key generator $G$ and encryption $E$ use independent sources of randomness. Then success probability of $R^{A}$ is:

$$
\begin{aligned}
& \operatorname{Pr}\left[R^{A}\left(E_{p k}(m)\right)=m\right] \\
= & \frac{1}{2} \operatorname{Pr}\left[R^{A}\left(E_{p k}(1)\right)=1\right]+\frac{1}{2} \operatorname{Pr}\left[R^{A}\left(E_{p k}(0)\right)=0\right] \\
= & \frac{1}{2}\left(\frac{1}{k} \operatorname{Pr}\left[A\left(E^{k}(1)\right)=1\right]+\sum_{i=2}^{k} \frac{1}{k} \operatorname{Pr}\left[A\left(E^{(i-1)}(0), E^{k-(i-1)}(1)\right)=1\right]\right) \\
& \quad+\frac{1}{2}\left(\sum_{i=1}^{k-1} \frac{1}{k} \operatorname{Pr}\left[A\left(E^{i}(0), E^{k-i}(1)\right)=0\right]+\frac{1}{k} \operatorname{Pr}\left[A\left(E^{k}(0)\right)=0\right]\right) \\
= & \frac{1}{2 k}\left(\operatorname{Pr}\left[A\left(E^{k}(1)\right)=1\right]+\operatorname{Pr}\left[A\left(E^{k}(0)\right)=0\right]\right)+\frac{k-1}{k} \cdot \frac{1}{2} \\
> & \frac{1}{2 k} \cdot 2\left(\frac{1}{2}+\epsilon\right)+\frac{k-1}{k} \cdot \frac{1}{2}=\left(\frac{1}{2 k}+\frac{\epsilon}{k}\right)+\left(\frac{1}{2}-\frac{1}{2 k}\right)=\frac{1}{2}+\epsilon / k,
\end{aligned}
$$

where probability is taken over uniform choice of one-bit message $m$, randomness of key generations $p k \leftarrow G\left(1^{n}\right)$ and $p k_{i} \leftarrow G\left(1^{n}\right)$, randomness of encryptions $E_{p k}(m), E_{p k_{i}}(0)$ and $E_{p k_{i}}(1)$ and over randomness of adversaries $A$ and $R$.

Lemma 5.3. If $(G, E, D)$ is $\left(\frac{1}{2}+\delta(n)\right)$-correct, then $(G, E, D)^{k(n)}$ is (1$\left.e^{-\delta^{2}(n) k(n) / 2}\right)$-correct.

Proof. Recall that $D^{k(n)}$ decodes the encrypted message $M_{1}, \ldots M_{k(n)}$, where $M_{i}=E_{p k_{i}}(m)$, by taking majority of outcomes $D_{s k_{i}}\left(M_{i}\right)$. The key pairs $\left(p k_{i}, s k_{i}\right)$ $\leftarrow G\left(1^{n}\right)$ are generated independently, therefore, applying the Chernoff bound, we estimate the probability of error of the encryption scheme $(G, E, D)^{k(n)}$ on a message $m$ as

$$
\operatorname{Pr}\left[\sum X_{i} \leq k / 2\right]=\operatorname{Pr}\left[\sum X_{i} \leq(1 / 2+\delta) k-\delta k\right]<e^{-\frac{\delta^{2} k^{2}}{2 k}}=e^{-\frac{\delta^{2} k}{2}}
$$

where $X_{i}$ indicates whether $D_{s k_{i}}\left(E_{p k_{i}}(m)\right)=m$.

${ }^{3}$ For brevity, we omit parameter $n$ and write $k$ instead of $k(n)$ and $\epsilon$ instead of $\epsilon(n)$. 
Now we have a method of provably secure correctness amplification that we will employ in the completeness proof of a public-key encryption scheme that we present in the next section.

\section{A Complete Public Key Encryption Scheme}

Before giving a proof of Theorem 3.2, we prove a lemma that allows us to simplify the proof of our encryption scheme's completeness:

Lemma 6.1. Every scheme $S \in \mathbf{P K C S}$ is reducible to some scheme $S^{\prime} \in \mathbf{P K C S}$, every algorithm of which runs in time $n^{2}$.

Every encryption scheme $S^{\prime} \in \mathbf{P K C S}$, every algorithm of which runs in time $n^{2}$, is reducible to some $\left(1-e^{-n}\right)$-correct encryption scheme $S^{\prime \prime} \in \mathbf{P K C S}$, every algorithm of which runs in time $n^{4}$.

Proof. The first part of our lemma is proved by padding argument. Assume that $S$ works in time $n^{k}$. Then, for a given security parameter $1^{n^{\prime}}, S^{\prime}$ simulates $S$ with security parameter $1^{n}, n=\left\lfloor\left(n^{\prime}\right)^{1 / k}\right\rfloor$. Obviously, this simulation can be done in time $\left(n^{\prime}\right)^{2}$. Note that, since the correctness $2 / 3$ is a constant, it does not decrease when we apply this argument.

Also, it is straightforward to construct a reduction $R$ from $S$ to $S^{\prime}$. For a given security parameter $n, R$ uses a provided black-box $A$ (that breaks $S^{\prime}$ ) with some security parameter $n^{\prime}, n^{k} \leq n^{\prime}<(n+1)^{k}$. The best value of this parameter can be found by testing the probability which $A$ stands at breaking $S^{\prime}$ for various values of $n^{\prime}$ from this interval. Again, Chernoff inequality is useful for this testing.

In order to prove the second part, it is sufficient to take $S^{\prime \prime}=\left(S^{\prime}\right)^{72 n+1}$ by Lemma 5.2 and Lemma 5.3.

\subsection{The Construction and Proof of Correctness}

Our complete public-key encryption scheme $\mathcal{S}=(\mathcal{G}, \mathcal{E}, \mathcal{D})$ is given below in Figure 6.1. For the correctness test, we choose $\delta=\frac{1}{2} \cdot\left(\left(1-\frac{1}{4 n}\right)+\left(1-\frac{1}{12 n}\right)\right)=1-\frac{1}{6 n}$, therefore $\Delta=\frac{1}{12 n}$. Also we choose $k=n^{4}$ (see Proposition 4.2). Time limit $T$ is set to $n^{4}$ (see Lemma 6.1 ; it coincides with $k$ only by chance).

Obviously, it is an encryption scheme (every algorithm of which runs in polynomial time). Further, it is $2 / 3$-correct, because any encryption scheme that is not $\left(1-\frac{1}{4 n}\right)$-correct passes the correctness test with probability at most $e^{-\frac{\Delta^{2} \cdot k}{2}}=e^{-\frac{n^{2}}{288}}$ by Proposition 4.2. Further, for all sufficiently large security parameters $n$, the set $I$ is guaranteed to be nonempty, because the identity encryption (the one that leaves message as it is) always passes the test.

\subsection{Proof of Completeness}

In order to prove the completeness of $\mathcal{S}$, assume that we have an encryption scheme $S_{j^{\prime}} \in$ PKCS. By transitivity of reductions and Lemma 6.1, it is reducible 


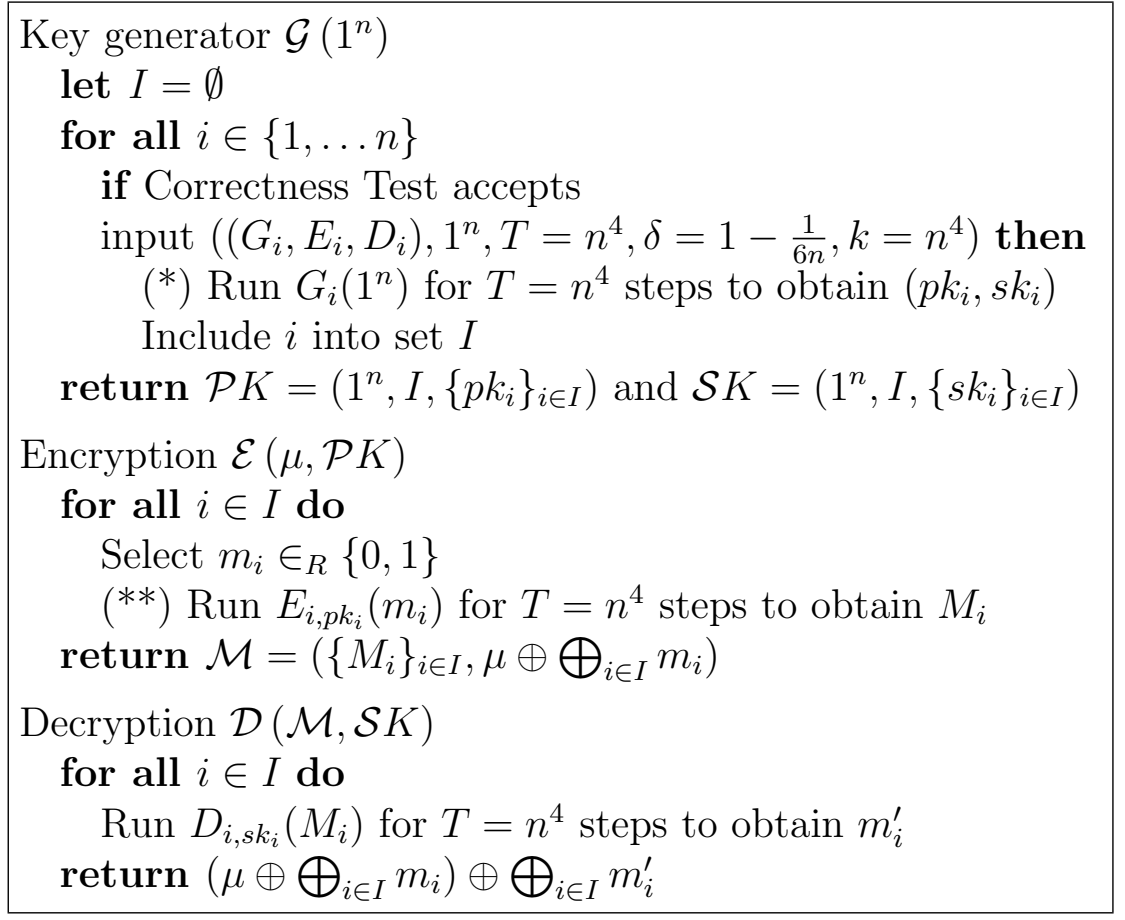

Figure 6.1

to some $\left(1-e^{-n}\right)$-correct public-key encryption scheme $S_{j}=\left(G_{j}, E_{j}, D_{j}\right)$, every algorithm of which runs in time $n^{4}$. It remains to construct a reduction from $S_{j}$ to $\mathcal{S}$.

We define a reduction $R$ on input composed of a security parameter $1^{n}$, a public key $p k$, and a codeword $M=E_{j, p k}(m)$, where $m \in_{R}\{0,1\}$. Note that for any security parameters $n \geq j$, the encryption scheme $S_{j}$ is among the first $n$ encryption schemes that are combined in $\mathcal{S}$.

1. Simulate key generator $\mathcal{G}$ on input $1^{n}$ to obtain a public key $\mathcal{P} K$. The only one exception is that, instead of generating $p k_{j}$ in step $\left(^{*}\right), R$ selects $p k_{j}$ to be equal to $p k$ (if occasionally $S_{j}$ fails the correctness test and thus $j \notin I$, then give up). All the other $\left\{p k_{i}\right\}_{i \neq j}$, are generated by $\mathcal{G}$.

2. Simulate encryption $\mathcal{E}$ on input composed of a new uniformly selected message $\mu \in_{R}\{0,1\}$ and of the public key $\mathcal{P} K$ in order to obtain a codeword $\mathcal{M}$ with the following modification: instead of computing $M_{j}=E_{j, p k}\left(m_{j}\right)$ in step (**), $R$ selects $M_{j}$ to be equal to $M$. Nonetheless, $m_{j}$ is generated (think of it as of $R$ 's guess of $m$ ) as well as all the other $\left\{m_{i}\right\}_{i \neq j}$. Then return ciphertext $\mathcal{M}=\left(\left\{M_{i}\right\}_{i \in I \backslash\{j\}} \cup\{M\}, \mu \oplus \bigoplus_{i \in I} m_{i}\right)$.

3. $\quad$ Provide oracle $A$ with security parameter $1^{n}$, public key $\mathcal{P} K$ and ask it to break the codeword $\mathcal{M}$. Output $A_{\mathcal{P} K}\left(1^{n}, \mathcal{M}\right) \oplus \mu \oplus m_{j}$.

By Definition 2.3, the probability that $R^{A}$ succeeds is exactly

$$
\operatorname{Pr}\left[R_{p k}^{A}\left(1^{n}, E_{j, p k}(m)\right)=m\right] \quad \text { for } p k \leftarrow G_{j}\left(1^{n}\right)
$$


where probability is taken over a uniformly selected one-bit message $m$, over randomness of key generation $G_{j}\left(1^{n}\right)$, encryption $E_{j, p k}(m)$, reduction $R_{p k}$ and over randomness of the invocation of probabilistic black-box $A$.

Conditioned on the event that $j \in I$, this probability, by the definition of reduction $R$ and by the definition of cryptosystem $\mathcal{S}=(\mathcal{G}, \mathcal{E}, \mathcal{D})$, is equal to

$$
\operatorname{Pr}\left[A_{\mathcal{P} K}\left(1^{n}, \mathcal{E}_{\mathcal{P} K}\left(\mu \oplus m_{j} \oplus m\right)\right)=m \oplus \mu \oplus m_{j}\right] \quad \text { for } \mathcal{P} K \leftarrow \mathcal{G}\left(1^{n}\right),
$$

where probability is taken over uniformly selected one-bit messages $m, \mu$ and $m_{j}$, over randomness of key generation $\mathcal{G}\left(1^{n}\right)$, encryption $\mathcal{E}_{\mathcal{P} K}$ and probabilistic blackbox $A$. Notice that key generation $p k \leftarrow G_{j}\left(1^{n}\right)$ and encryption $E_{j, p k}(m)$ become parts of key generation $\mathcal{P} K \leftarrow \mathcal{G}\left(1^{n}\right)$ and encryption $\mathcal{E}_{\mathcal{P} K}$ correspondingly.

Let $\tilde{\mu}=\mu \oplus m_{j} \oplus m$. Then the probability above is equal to

$$
\operatorname{Pr}\left[A_{\mathcal{P} K}\left(1^{n}, \mathcal{E}_{\mathcal{P} K}(\tilde{\mu})\right)=\tilde{\mu}\right] \quad \text { for } \mathcal{P} K \leftarrow \mathcal{G}\left(1^{n}\right),
$$

where probability is taken over a uniformly selected one-bit message $\tilde{\mu}$, over randomness of key generation $\mathcal{G}\left(1^{n}\right)$, encryption $\mathcal{E}_{\mathcal{P} K}$ and probabilistic black-box $A$. This is exactly the success probability of adversary $A$.

To finish the proof, it remains to notice that the encryption scheme $S_{j}=\left(G_{j}, E_{j}\right.$, $D_{j}$ ) passes the correctness test with probability greater than $1-e^{-n}$, thus $j \notin I$ with a negligible probability.

Acknowledgements. We thank Oded Goldreich for discussion on universal primitives that are implied from Levin's one-way function. We are grateful to Sergey Nikolenko for fruitful discussions on early stages of this work. Also, we would like to thank Alexander Shen and Nikolay Vereshchagin for their helpful comments on this paper.

\section{References}

[1] M. Ajtai, C. Dwork: A public-key cryptosystem with worst-case/average-case equivalence, in: ACM Symposium on Theory of Computing (STOC, El Paso, 1997), ACM, New York (1999) 284-293.

[2] C. Dwork, M. Naor, O. Reingold: Immunizing encryption schemes from decryption errors, in: Advances in Cryptology (EUROCRYPT, Interlaken, 2004), C. Cachin et al. (ed.), Springer, Berlin (2004) 342-360.

[3] L. Fortnow, R. Santhanam: Hierarchy theorems for probabilistic polynomial time, in: IEEE Symposium on Foundations of Computer Science (FOCS, Rome, 2004), IEEE Computer Society, Washington (2004) 316-324.

[4] S. Goldwasser, M. Bellare: Lecture Notes on Cryptography, Summer course on cryptography, MIT, Cambridge (2001).

[5] O. Goldreich, S. Goldwasser, S. Halevi: Eliminating decryption errors in the AjtaiDwork cryptosystem, in: Advances in Cryptology (CRYPTO, Santa Barbara, 1997), B. S. Kaliski jun. (ed.), Springer, Berlin (1997) 105-111. 
[6] S. Goldwasser, S. Micali: Probabilistic encryption, J. Comput. Syst. Sci. 28 (1984) 270-299.

[7] O. Goldreich: Foundations of Cryptography: Volume 1, Basic Tools, Cambridge University Press, Cambridge (2001).

[8] O. Goldreich, D. Ron: On universal learning algorithms, Inf. Process. Lett. 63 (1997) 131-136.

[9] D. Harnik, J. Kilian, M. Naor, O. Reingold, A. Rosen: On robust combiners for oblivious transfer and other primitives, in: Advances in Cryptology (EUROCRYPT, Aarhus, 2005), R. Cramer (ed.), Springer, Berlin (2005) 96-113.

[10] T. Holenstein: Key agreement from weak bit agreement, in: ACM Symposium on Theory of Computing (STOC, Baltimore, 2005), H. N. Gabow et al. (ed.), ACM, New York (2005) 664-673.

[11] J. Hoffstein, J. Pipher, J. Silverman: NTRU: A ring-based public key cryptosystem, in: Algorithmic Number Theory Symposium (ANTS, Portland, 1998), J. P. Buhler (ed.), Springer, Berlin (1998) 267-288.

[12] T. Holenstein, R. Renner: One-way secret-key agreement and applications to circuit polarization and immunization of public-key encryption, in: Advances in Cryptology (CRYPTO, Santa Barbara, 2005), V. Shoup (ed.), Springer, Berlin (2005) 478-493.

[13] R. Impagliazzo, S. Rudich: Limits on the provable consequences of one-way permutations, in: ACM Symposium on Theory of Computing (STOC, Santa Barbara, 1988), Springer, Berlin (1990) 8-26.

[14] L. Levin: One-way functions and pseudorandom generators, Combinatorica 7 (1987) 357-363. 